http://jmscr.igmpublication.org/home/ ISSN (e)-2347-176x ISSN (p) 2455-0450

crossref DOI: https://dx.doi.org/10.18535/jmscr/v9i12.01

\title{
The Extent to Which Diclofenac is used to Treat Fever Cases of Children and the Elderly in the Emergency Department of Governmental and Private Hospitals in Amman
}

\author{
Author \\ Jafar Mohammad Ahmad Yasin \\ Accident and Emergency Medicine Specialist
}

\begin{abstract}
This study was conducted to investigate the rate of usage of Diclofenac in the treatment of children and the elderly by doctors compared to other fever treatment drugs in the EDs of governmental and private hospitals in Amman- Jordan.

Procedures: A survey of 126 patients in the 12-60 age groups who attended to hospitals EDs to get treatment for fever cases and investigated the use of Diclofenac treatment in the treatment of these cases or other fever treatment drugs were used, from August 2021 to October 2021 in governmental and private hospitals in Amman- Jordan.

Results: In this study, the usage of Diclofenac in the treatment of fever cases was noticed at an rate of $89 \%$ compared to other fever treatment drugs in private hospitals, and of average of $38 \%$ compared to other fever treatment drugs in governmental hospitals, due to the financial expenses of the drug compared to other fever treatment drugs and the amount of quantities available for usage in the hospital itself.

Conclusion: The researcher recommends expanding the scope of the study sample to study and analyze a larger sample, which will lead to better access to Diclofenac and greater satisfaction scores than patients attending treatment in the emergency departments of these hospitals.
\end{abstract}

\section{Introduction}

Diclofenac is one of the strongest analgesics and heat-reducers for children, and there is a significant use rate among mothers, the elderly and even among doctors themselves who prefer this type as a strong, heat-reducing analgesic in pursuit of a faster drug response.

Diclofenac is a nonsteroidal anti-inflammatory drug of the phenylacetic acid class with antiinflammatory, analgesic, and antipyretic properties. Contrary to the action of many traditional nonsteroidal anti-inflammatory drugs, diclofenac prevents cyclooxygenase (COX)-2 enzyme with larger potency than it does COX-1. Similar to other nonsteroidal anti-inflammatory drugs, diclofenac is associated with serious dosedependent gastrointestinal, cardiovascular, and renal adverse effects.

Since the introduction in 1973, a number of different diclofenac-containing drug products have been developed with the goal of improving efficacy, tolerability, and patient treatment satisfaction.

Delayed- and extended-release forms of diclofenac sodium were at the start developed with the goal of improving the safety profile of 
diclofenac and providing convenient, once-daily dosing for the treatment of patients with chronic pain. New drug products consisting of diclofenac potassium salt were associated with faster absorption and rapid onset of pain relief.

These include diclofenac potassium liquid-filled soft gel capsules, diclofenac potassium powder for oral solution, and diclofenac potassium immediate-release tablets.

The creation of topical formulations of diclofenac while minimizing systemic absorption, its allows local treatment of pain and inflammation of diclofenac. Solu Matrix diclofenac, consisting of submicron particles of diclofenac free acid and a proprietary combination of excipients, was developed to provide analgesic efficacy at reduced doses associated with lower systemic absorption. This review illustrates how pharmaceutical technology has been used to modify the pharmacokinetic properties of diclofenac, leading to the creation of novel drug products with improved clinical utility.

And this study will investigate the rate at which diclofenac is used in the treatment of children and the elderly by doctors to other fever treatment drugs, in both governmental and private EDs hospitals in Amman- Jordan district and compare their usage rates and the satisfaction of patients of their case treatment.

\section{General Properties of Diclofenac}

Diclofenac belongs to a group of nonsteroidal anti-inflammatory drugs, that inhibit both COX-1 and COX-2 enzymes. The binding of nonsteroidal anti-inflammatory drugs to $\mathrm{COX}$ isozymes inhibits the synthesis of prostanoids (i.e., prostaglandin [PG]-E2, PGD2, PGF2, prostacyclin [PGI2], and thromboxane [TX] A2). PGE2 is the dominant prostanoid produced in inflammation, and what believed to be the main mechanism of the potent analgesic and anti-inflammatory properties of these agents, is the inhibition of its synthesis by nonsteroidal anti-inflammatory drugs.

Paola Patrignani and colleagues developed The human whole blood in vitro assay has been used to measure the degree of selectivity of nonsteroidal anti-inflammatory drugs toward COX-2 or COX-1 by quantifying the inhibition of COX-2 - dependent formation of PGE2 produced by monocytes, following lipopolysaccharide (LPS) stimulation versus COX-1-dependent formation of TXB2, the non-enzymatic hydrolysis product of TXA2 produced by platelets. Although diclofenac is commonly referred to as a traditional nonsteroidal anti-inflammatory drug in the published literature, these assays have demonstrated that it has a higher selectivity for COX-2 than for COX-1, in contrast with most traditional nonsteroidal anti-inflammatory drugs. The degree of COX-2 selectivity demonstrated for diclofenac is comparable to that of celecoxib[51]. Diclofenac is more potent in inhibiting COX-2 than COX-1 isoenzymes. However, the estimated IC50 (concentration causing $50 \%$ inhibition of activity) values for COX-1 and COX-2 of different COX inhibitors has been shown to vary between models, and selectivity is dose dependent in some cases.

So Diclofenac is essentially not preferred for young children, and is used only in conditions necessary for high degree fever, although that's it's not preferred, the age group used to treat their fever cases with Diclofenac can be limited to over 12 years old, but if there is a medical necessity the drug can be prescribed by a doctor between the ages of 3 and 12, while the age group under 3 years old is not recommended to use Diclofenac.

As for the symptoms of overdose of Diclofenac for children headaches, nausea, vomiting, severe pain in the mouth of the stomach, and may lead to gastrointestinal bleeding. Sometimes it develops to include: diarrhea, dizziness, tinnitus, coma as well as occasional convulsions. Very high-dose poisonings cause severe and sudden kidney function failure and destruction of liver cells.

\section{Materials and Methods}

A survey of 126 randomly selected patients under two specific criteria was conducted which are first is the (12-60) years old age group, second is who 
attended treatment for fever treatments and investigated the use of Diclofenac in the treatment of these cases or other fever treatments were used in these hospitals EDs, from August 2021 to October 2021 in governmental and private hospitals in Amman- Jordan.

Interviews were also conducted with emergency doctors who treated the selected individuals about the determinants of the use of Diclofenac or other fever treatments for these cases, and patient satisfaction with the procedures followed and the results achieved in relation to their fever cases were also reviewed.

\section{Ethical Issues}

An official coverage for the research was issued by the Jordanian Ministry of Health to conduct this study and each patient case was examined in complete secrecy without expressing the patient's identity and gender, as well as the identities of the doctors interviewed and taken their opinions.

\section{Results}

Of the 126 patients examined, 62 (49\%) were checked in governmental hospitals EDs for the specific scope of the study to treat fever cases, and $64(51 \%)$ were patients who were checked in private hospitals EDs for the specific scope of the study to treat fever cases.

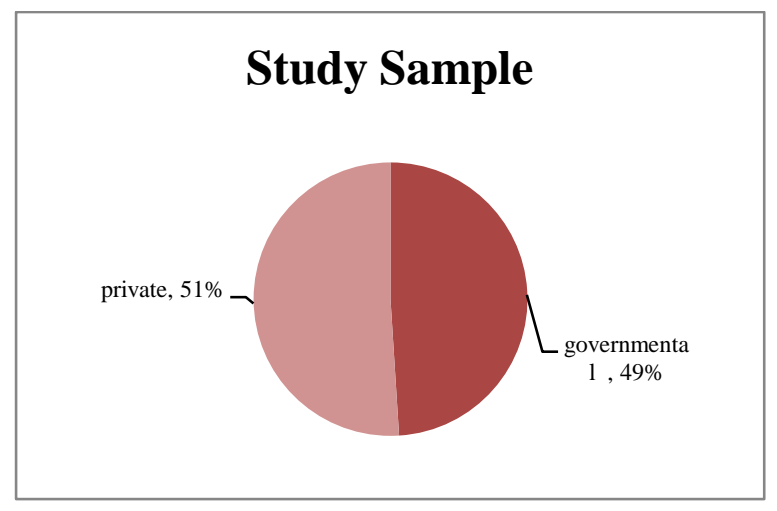

Diclofenac was used to treat fever cases in government hospitals with 24cases (38\%) of patients, patients expressed satisfaction scores as the following: 33 cases (53\%) expressed low satisfaction, 18cases (29\%) expressed moderate satisfaction, and 11 cases (18\%) expressed a high satisfaction score.

Diclofenac was used to treat fever cases in private hospitals with 57 cases $(89 \%)$ of patients, patients expressed satisfaction scores as the following: 4 cases $(6 \%)$ expressed a low satisfaction score, 20cases $(31 \%)$ expressed moderate satisfaction, and 40cases (63\%) expressed a high satisfaction score.

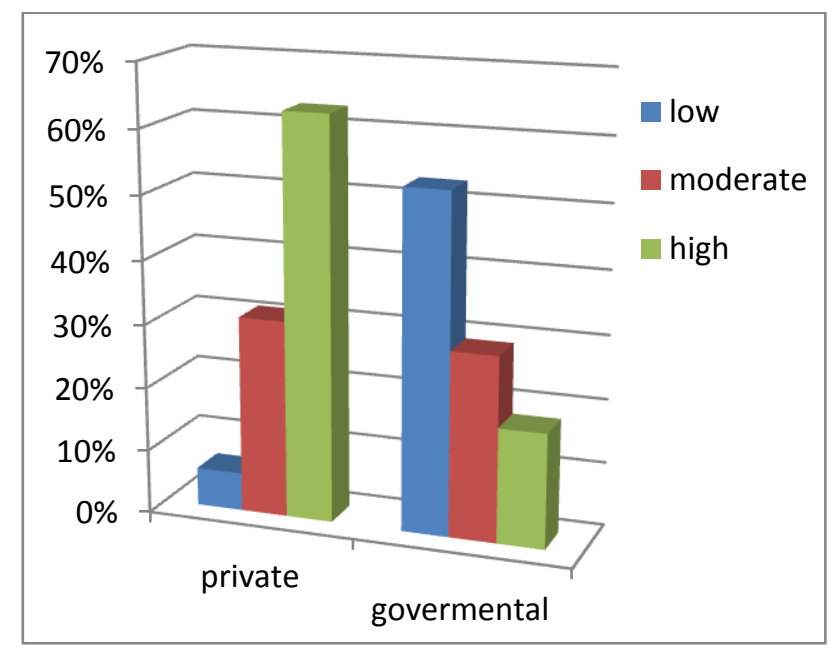

And with regard to the interviews of the doctors treating these cases, the doctors instructed, one of the determinants of the usage of the drug is the quantities available for use and the financial expenses of the drug Diclofenac itself compared to other fever treatment drugs.

\section{Discussion}

Governmental hospitals are experiencing 38\% less usage of Diclofenac than other fever treatment drugs, due to the limited amounts of the Diclofenac for treatment and the financial expenses of the drug, expressed by the doctors supervising these cases, resulting in low satisfaction rates by patients treated with them.

On the other hand, private hospitals are experiencing a $89 \%$ greater use of Diclofenac than other fever treatment drugs, due to the high quantities that are available to those hospitals, and the high ability to cover the financial expenses of the drug, as expressed by the doctors supervising these cases, resulting in high satisfaction rates by patients treated. 


\section{Conclusion}

The researcher recommends A larger sample size is needed to further analyze, which will lead to better access to objective information of the usage of Diclofenac which will help is conducting greater road map to rise satisfaction scores of patients attending treatment their fever cases in the EDs of the hospitals.

\section{References}

1. Vane JR. Inhibition of prostaglandin synthesis as a mechanism of action for aspirin-like drugs. Nat N Biol. 1971;231(25):232-5.

2. Ku EC, Lee W, Kothari HV, Scholer DW. Effect of diclofenac sodium on the arachidonic acid cascade. Am J Med. 1986;80(4B):18-23.

3. Patrono C, Patrignani P, Garcia Rodriguez LA. Cyclooxygenase-selective inhibition of prostanoid formation: transducing biochemical selectivity into clinical readouts. J Clin Invest. 2001;108(1):7-13.

4. Smyth EM, Grosser T, Wang M, Yu Y, Fitz Gerald GA. Prostanoids in health and disease. J Lipid Res. 2009;50 (Suppl): S423-8.

5. Grosser T, Fries S, FitzGerald GA. Biological basis for the cardiovascular consequences of $\mathrm{COX}-2$ inhibition: therapeutic challenges and opportunities. $\mathrm{J}$ Clin Invest. 2006;116(1):4-15.

6. Patrignani P, Panara MR, Greco A, Fusco O, Natoli C, Iacobelli S, et al. Biochemical and pharmacological characterization of the cyclooxygenase activity of human blood prostaglandin endoperoxide synthases. J Pharmacol Exp Ther. 1994;271(3):1705-12.

7. Patrono $\mathrm{C}$, Ciabattoni $\mathrm{G}$, Pinca $\mathrm{E}$, Pugliese F, Castrucci G, De Salvo A, et al. Low dose aspirin and inhibition of thromboxane B2 production in healthy subjects. Thromb Res. 1980;17(3-4):317-27.
8. Huntjens D, Danhof M, Della Pasqua O. Pharmacokinetic-pharmacodynamic correlations and biomarkers in the development of COX-2 inhibitors. Rheumatology. 2005;44(7):846-59.

9. Garcia Rodriguez LA, Tacconelli S, Patrignani P. Role of dose potency in the prediction of risk of myocardial infarction associated with nonsteroidal antiinflammatory drugs in the general population. J Am Coll Cardiol. 2008;52(20):1628-36.

10. Warner TD, Giuliano F, Vojnovic I, Bukasa A, Mitchell JA, Vane JR. Nonsteroid drug selectivities for cyclooxygenase-1 rather than cyclo-oxygenase2 are associated with human gastrointestinal toxicity: a full in vitro analysis. Proc Natl Acad Sci. 1999;96(13):7563-8.

11. Altman, R., Bosch, B., Brune, K. et al. Advances in NSAID Development: Evolution of Diclofenac Products Using Pharmaceutical Technology. Drugs 75, 859-877 (2015). 